\title{
Ten years of Fermi LAT observations and the new 4FGL and 4LAC catalogs
}

\author{
Stefano Ciprini ${ }^{* \dagger}$ \\ Space Science Data Center, Agenzia Spaziale Italiana (SSDC-ASI), I-00133, Roma, Italy \\ Istituto Nazionale di Fisica Nucleare (INFN), Sezione di Roma Tor Vergata, I-00133, Roma, Italy \\ E-mail: stefano.cipriniessdc.asi.it
}

\begin{abstract}
After the first ten years of Fermi Gamma-ray Space Telescope mission, both the Large Area Telescope (LAT) and the Gamma-ray Burst Monitor (GBM) instruments have better performance than ever, with enhancements to pipeline processing and analysis software. This is promising for new and relevant science and discoveries to be accomplished with the Fermi mission, in particular for high-energy and multi-frequency astrophysics, and for astro-particle multi-messenger physics. This in view of synergetic observations in parallel to the new and incoming observatories and experiments for cosmic photons, gravitational waves, neutrinos and ultra-high-energy cosmic rays. The Fermi LAT collaboration has released, at the end of February 2019, an early version of the fourth Fermi Gamma-ray LAT (4FGL) source catalog, based on the first eight years of LAT science data collection. The 4FGL catalog is the largest and deepest ever in gamma-ray photon energy range (from $50 \mathrm{MeV}$ to $1 \mathrm{TeV}$ ) and represents one of the main LAT goals after the first 10 years of Fermi operations. The 4FGL catalog includes 5098 gamma-ray sources detected above 4 sigma significance, for which localization and spectral properties are provided. Additional information and products will be added in subsequent updates, including light curves, analysis flags, more or revised information about multi-wavelength associations of some sources. The companion fourth Fermi LAT AGN catalog (4LAC), composed of more than 3100 sources, is focussed on blazars and other AGN, objects representing more than $70 \%$ of all the high-Galacticlatitude gamma-ray sources detected in the 4FGL catalog. The 4LAC catalog includes information on AGN source or candidate associations, basic multi-frequency properties, source class, synchrotron peak, redshifts, gamma-ray spectral and variability properties, and characterization of the whole gamma-ray AGN population.
\end{abstract}

Accretion Processes in Cosmic Sources - II - APCS2018

3-8 September 2018

Saint Petersburg, Russian Federation

\footnotetext{
*Speaker.

† on behalf of the Fermi-LAT collaboration
} 


\section{Fermi Large Area Telescope instrument}

Gamma-ray astrophysics is relatively young and many basic discoveries are still being made. Gamma rays cover a wide interval of the electromagnetic spectrum, and the gamma-ray sky is relatively poorly studied. Medium and high-energy ( $\mathrm{MeV}-\mathrm{GeV}$ photon energy) gamma rays probe the non-thermal universe and the extreme environments hosting powerful acceleration of cosmic particles, and violent and variable astrophysical multi-messenger physical systems. High energy gamma-ray astronomy above about $50 \mathrm{MeV}$ photon energy started with early NASA SAS-2 (Nov. 1972 - Jun. 1973) [16] and ESA COS-B (Aug. 1975 - Apr. 1982) [14] space satellites that led, almost ten years later, to the EGRET instrument [21] on board of the NASA Compton GammaRay Observatory (CGRO) (Apr. 1991 - Jun. 2000). In the new century, on Apr. 23, 2007 the compact Agile satellite was launched by ASI [19]. About 400 days later the NASA probe class mission named Fermi Gamma-Ray Space Telescope ${ }^{12}$, formerly known as the Gamma-ray Large Area Space Telescope (GLAST), was successfully launched in June 11, 2008 at 12:05 PM EDT (16:05 UTC) on United Launch Alliance Delta II-Heavy 7920-H rocket vehicle from the NASA Kennedy Space Center, Cape Canaveral Air Force Station, USA.

The Fermi Gamma-ray Space Telescope is a powerful high-energy space observatory, a NASA probe-class mission, characterized by huge leap in all key capabilities, with performance unmatched by any existing or planned ground and space observatory for $E>100 \mathrm{MeV}$ gamma-ray astronomy. The Fermi mission is working to unveil the mysteries of the gamma-ray universe, and has been built thanks to an international effort bringing together the astrophysics and high-energy particle physics communities. The Fermi Gamma-ray Space Telescope was developed by NASA in collaboration with the U.S. Department of Energy, along with important contributions from academic institutions and partners in France, Germany, Italy, Japan, Sweden, and the United States. The space mission named GLAST was rechristened in Aug. 2008 for the physicist Enrico Fermi, reflecting the ongoing mysteries of the gamma-ray sky and the Italian contribution to the design, construction, simulation of the LAT instrument, especially the contribution of the Italian Institute for Nuclear Physics (INFN). INFN is, indeed, an institute created in 1951 to develop the scientific tradition started with Enrico Fermi and his group in Rome on '1930s with the experimental and theoretical researches on nuclear physics. Enrico Fermi, who won the 1938 Nobel Prize in Physics, is best known for building the first man-made nuclear reactor in 1942, and was a brilliant theoretical physicist as well, being also the first to propose a mechanism by which powerful cosmic objects could accelerate particles (also electrons used in blazar jet high-energy emission models) strongly enough to generate gamma rays.

- The first order Fermi acceleration is diffusive shock acceleration, an efficient method for accelerating particles in supernova remnant shock waves, with velocity vectors randomized upstream and downstream of the shock (for example scattering of cosmic rays by Alfven waves).

- The second order Fermi acceleration (already suggested in 1949) is particle acceleration via statistical gain of energy through collisions with interstellar clouds.

The Fermi mission satellite studies energetic gamma-rays, observing physical processes far beyond the capabilities of earthbound laboratories and opening a wide electromagnetic energy

\footnotetext{
${ }^{1}$ fermi.gsfc.nasa.gov

$2_{\text {www.nasa.gov/fermi }}$
} 
window on the universe. The Fermi satellite was set initially on a circular orbit, at $565 \mathrm{~km}$ altitude with $25.6^{\circ}$ inclination, operating in an all-sky survey primary mode with the scan of the entire sky every 3 hours. Autonomous Repoint Request (ARR) and Target of Opportunity (ToO) pointed observations are additional operating modes. The two Fermi instruments are:

- the Large Area Telescope (LAT) ${ }^{3}$, a pair tracker-converter telescope allowing to observe $20 \%$ of sky at any instant at $\gamma$-ray energies between $20 \mathrm{MeV}$ and above $300 \mathrm{GeV}$, with absolute event timing precision up to $300 \mathrm{~ns}$ [11];

- the gamma-ray Burst Monitor (GBM) ${ }^{4}$ devoted to correlative GRB and transient observations, over the whole Earth-unocculted sky, in the energy range between about $8 \mathrm{keV}$ and $40 \mathrm{MeV}$, with absolute timing precision of $2 \mu \mathrm{s}$.

This space telescope can therefore cover eight orders of magnitude in energy ( $\sim 8 \mathrm{keV}$ to $\sim 2 \mathrm{TeV}$ ), surveying the entire gamma-ray sky every three hours. The Fermi spacecraft has also on-board GPS, a Ku-band antenna for data transmission via the Tracking and Data Relay Satellite System (TDRSS, with 40 Mbps rate) and a propulsion system for collision avoidance and deorbiting. To take full advantage of the LAT's large field-of-view (FoV), the primary observing mode of Fermi observatory is the so-called all-sky "scanning" mode in which the normal to the front of the instrument ( $z$ axis) on alternate orbits is pointed to $+50^{\circ}$ from the zenith direction and towards the pole of the orbit and to $-50^{\circ}$ from the zenith on the subsequent orbit. In this way, after 2 orbits, about 3 hours for Fermi's orbit at $\sim 565 \mathrm{~km}$ and $25.5^{\circ}$ inclination, the sky exposure is almost uniform. For particularly interesting targets of opportunity, the observatory can be inertially pointed. There are originally four general Fermi observing modes and related instrument operations:

-1) the primary mode (sky survey) with all-sky scan every 3 hours;

- 2) the Autonomous Repoint Request (ARR) for GRB/transient following on-board software triggers;

- 3) Target of Opportunity (ToO) following GI proposals and opportunities to increase exposure towards a specific target;

- 4) modified survey profile (e.g. Galactic center biased from Dec. 2013 to Dec. 2014).

A new observing mode profile is implemented from Apr. 2018, with the resume of operations after the failure of a mechanism that drives one solar panel array and that triggered an automatic safe hold mode that powered down Fermi's instruments, as described in the next section.

Fermi LAT is an all-sky hunter and surveyor for high-energy transients and flares and all-sky monitor for variability of the restless, variable, violent, flaring and transient high-energy sky at gamma-ray energies above $100 \mathrm{MeV}$ [20]. From within its 1.8-meter cube housing, the Fermi LAT uses 880000 silicon microstrip detectors to detect high-energy gamma rays with unprecedented resolution and sensitivity, pushing new boundaries in gamma-ray astrophysics and multifrequency/multi-messenger astroparticle physics. The science objectives and results of the Fermi LAT instrument are:

- to determine the nature and physical processes of high energy gamma-ray sources, in particular resolving the gamma-ray sky and determining the origins of diffuse emission and unidentified sources nature;

\footnotetext{
${ }^{3}$ glast.sites.stanford.edu

${ }^{4}$ gammaray.nsstc.nasa.gov/gbm/
} 
- to make significant progress in $\mathrm{MeV}-\mathrm{GeV}$ astronomy, understanding the gamma-ray sky and the mechanisms of particle acceleration and radiation in astrophysical sources;

- to study the high-energy behavior of transients and gamma-ray bursts (GRBs), like the short GRB originated by merging binary neutron stars producing bursts of gravitational waves events in particular;

- to study and make significant progress for gamma-ray sources and science topics like: gammaray and multi-frequency/messenger analysis methods; gamma-ray source detection and catalogs; pulsars and pulsars wind nebulae; supermassive black-hole and jets of active galactic nuclei (AGN); energetic compact binary star systems and novae; diffuse gamma-ray emission of our Galaxy; supernova remnants; studies unassociated gamma-ray sources; searching for primordial intermediate-mass black hole evaporation; extragalactic gamma-ray background, extragalactic background light; high-redshift blazars and GRBs, solar flares, Moon emission and other potential solar system sources; terrestrial gamma-ray flashes;

- to study charged particles like cosmic-ray electrons and protons;

- to make significant progress in multi-wavelength astrophysics and multi-messenger astroparticle physics;

- to provide insights probing the early universe and make constraints, investigating some topics in fundamental physics and dark matter searches like indirect dark matter model constraints for example from non-astrophysical diffuse excesses emission in the Galactic center, emission in clusters of galaxies and in nearby dwarf spheroidal galaxies; searching evidences for axion like particles using distant blazars/GRBs/supernovae probes, searching for any signal and hint of potential new laws of fundamental physics.

Summarizing with the Fermi mission astrophysicists have a superior all-sky telescope to study the violent, variable, energetic objects and events of the high-energy Universe, while physicists are able to study subatomic particles at energies far greater than those seen in ground-based particle accelerators, and cosmologists are gaining useful information about the early evolution of the Universe. Following these goals the properties of the LAT includes:

- a good angular resolution for source localization and multi-wavelength/messenger studies;

- high sensitivity over a broad field-of-view to perform serendipitous discoveries during the sky survey with monitor of the temporal variability and increasing detections of transient objects/events;

- good calorimetry over an extended energy band allowing to study spectral breaks, curvatures, cut-offs and other features;

- good calibration and stability for absolute, long-term flux monitoring measurements.

The Fermi LAT instrument consists of four main components: 1) a pair-conversion telescope with a precision converter-tracker (silicon micro-strip detectors); 2) a calorimeter (CsI with PIN diode 


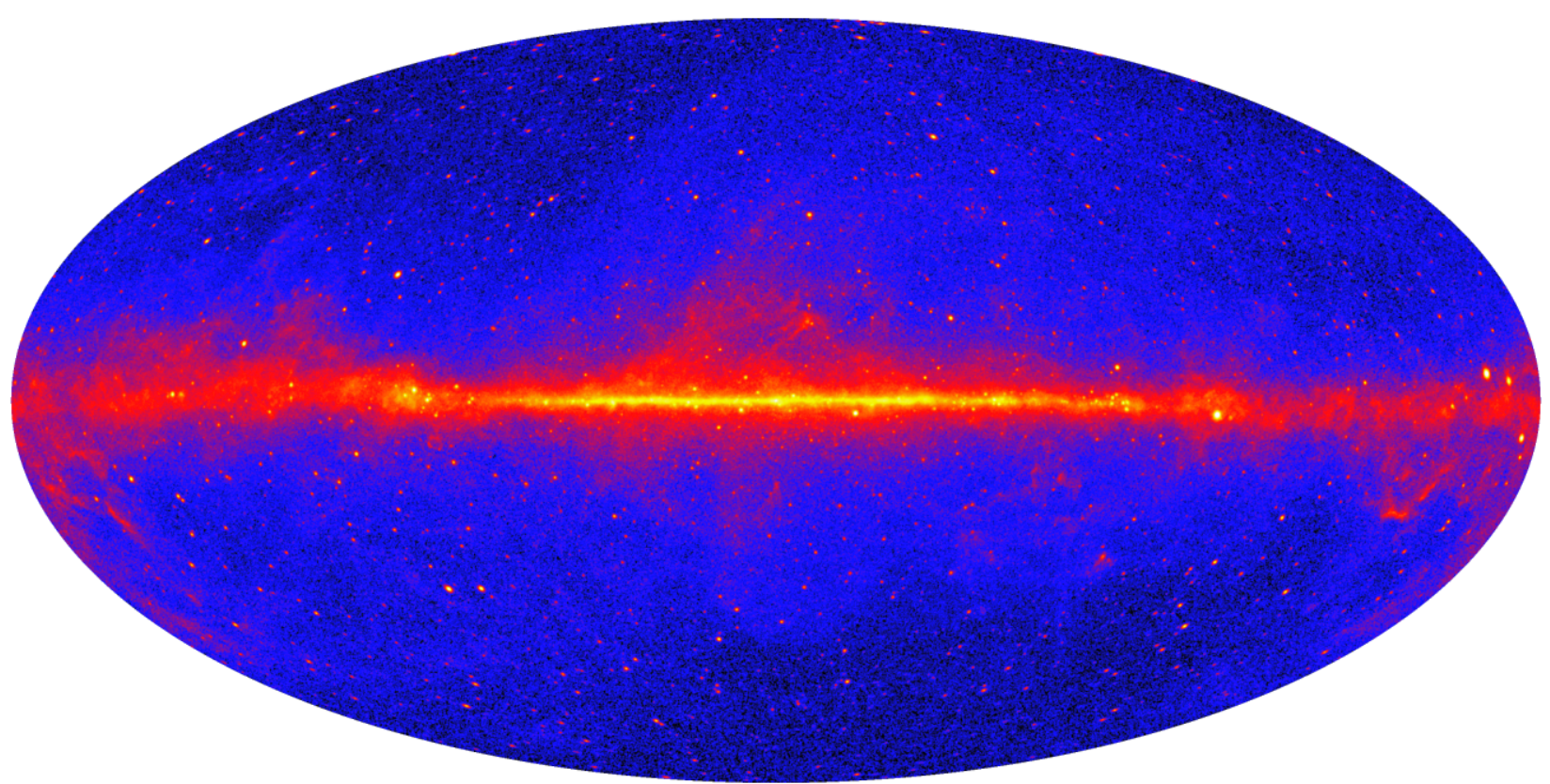

Figure 1: 10-year (August 4, 2008 - August 4, 2018) gamma-ray intensity all-sky image obtained by the Fermi LAT. The map is built using Pass 8 Source class PSF3 event type data, with intensity units, integrated events at E $>1 \mathrm{GeV}, 100$ deg zenith angle limit, Galactic coordinates with Hammer-Aitoff projection and logarithmic scaling. In the 10-year data set, the counts map contains 6873913 gamma-ray photons, and is smoothed with a $0.25 \mathrm{deg}$ FWHM Gaussian, representing intensity units, from about $3 \times 10^{-7}$ to $10^{-3} \mathrm{~cm}^{-2}$ $\mathrm{s}^{-1} \mathrm{sr}^{-1}$. Credits NASA/DOE/Fermi-LAT Collaboration.

readout); 3) a segmented anticoincidence plastic detector (ACD); 4) a data acquisition system (DAQ). The LAT is designed and assembled as a $4 \times 4$ array of 16 identical "tower" modules, with the converter-tracker part supported by a low-mass carbon-composite and vented aluminum honeycomb grid structure. These four subsystems work together measuring the directions, energies, and arrival times of gamma ray photons incident over a large $\mathrm{FoV}$, while rejecting background from cosmic rays. The modular implementation allowed the construction, at reasonable incremental cost of the fully functional LAT.

Incident high-energy gamma rays cannot be reflected or refracted; they interact undergoing pair-conversion into $e^{+} e^{-}$pairs, preferentially in a thin, high- $Z$ (tungsten) foil. The LAT measures these tracks and energy of the subsequent electromagnetic shower that develops in the calorimeter. The overall aspect ratio of the LAT converter-tracker (height/width) is 0.4 , allowing a large FoV and ensuring that nearly all $e^{+} e^{-}$pair-conversion events initiated in the tracker will pass into the calorimeter for energy measurement. The ACD covers the tracker array, and a programmable trigger and data acquisition system (DAQ) utilizes prompt signals available from the tracker, calorimeter, and ACD subsystems to form a trigger, where self-triggering capability is possible because of the choice of silicon-strip detectors (SSDs). The LAT SSDs are AC-coupled, with 384 56- $\mu \mathrm{m}$ wide aluminum readout strips spaced at $228 \mu \mathrm{m}$ pitch distance, and were produced on $n$-intrinsic $15-\mathrm{cm}$ wafers, with an area of $8.95 \times 8.95 \mathrm{~cm}^{2}$ each, with an inactive area $1 \mathrm{~mm}$ wide around the edges, and a thickness of $400 \mu \mathrm{m}$. The amount of detectors used for the LAT tracker subsystem, is equivalent to a total surface of about $83 \mathrm{~m}^{2}$ of silicon detectors (11500 SSDs), being second only to CMS at the Large Hadron Collider of CERN. 
Heat produced by the tracker, calorimeter, and DAQ electronics is transferred to radiators through heat pipes in the grid. Each tracker tower module has $18 x, y$ alternated tracking planes, consisting of 2 layers ( $x$ and $y$ ) of single-sided SSDs. Sets of 4 SSDs bonded edge to edge with epoxy and wire bonded strip to strip form "ladders". Each detector layer in a tracker module consists of 4 such ladders spaced apart by $0.2 \mathrm{~mm}$ gaps. The planes-support structure is a stack of 19 composite "trays" with low-mass structure also serving to conduct heat to the base of the tracker array. The probability distribution for the photon reconstructed direction from an astronomical point source is referred to as the Point Spread Function (PSF). Optimal results are obtained when the $e^{-}$ and $e^{+}$directions are measured immediately following the conversion, while multiple scattering of these and bremsstrahlung production limit the obtainable resolution. At $100 \mathrm{MeV}$ the penalty for missing one of the first hits is about a factor of two in resolution, resulting in large tails in the PSF. The LAT was designed balancing the need for thin converters, to achieve a good PSF at low energy, where the PSF is determined primarily by the $\sim 1 / E$ dependence of multiple scattering, versus the need for converter material to maximize the effective area, important at high energy: the tracker is hence divided into 2 regions, "front" (first $12 x, y$ tracking planes, 0.03 radiation lengths thick, to optimize low energy PSF) and "back" ( $4 x, y$ planes $\sim 6$ times thicker to maximize the effective area).

Each calorimeter module has $96 \mathrm{CsI}(\mathrm{Tl})$ crystals (arranged horizontally in 8 layers of 12 crystals each), and each layer is aligned $90^{\circ}$ with respect to its neighbors, forming an $x, y$ (hodoscopic) array, providing a total vertical depth of 8.6 radiation lengths. The total calorimeter depth is 10.1 radiation lengths. The ACD provides charged-particle background rejection, through plastic scintillator tiles that are segmented to suppress the backsplash (self-veto) effect created by the calorimeter mass at high energies. The DAQ collects data from the other subsystems, implements the multilevel event trigger, supplies on-board event processing to run filter algorithms to reduce the number of downlinked events, and provides an on-board science analysis platform to rapidly search for transients. A detailed Monte Carlo simulation of the LAT's response to signals (gamma rays) and backgrounds (cosmic-rays, albedo gamma rays, etc.) was developed and validated, representing an important contribution in the design and optimization of the LAT, and in reconstruction (tracks, events, classification, energy) and background rejection. More details of the LAT instrumentation, design, performance, calibration are presented, for example, in [13, 11, 3, 5].

\section{Fermi ten years of all-sky survey observations}

An instrument surveying the entire gamma-ray celestial sphere and monitoring all the timescales from fractions of seconds to years as the Fermi LAT can be conceived as a spatial-survey and timedomain monitor experiment that is essential to study cosmic particle accelerators in five dimensions in photon energy, time, sky position (R.A., Dec.) or spatial distribution, and in cosmic distance (redshift). The Fermi LAT all-sky survey means uniformity of sky coverage, enhanced opportunities for serendipitous discoveries and detection of transients, these also in the multi-wavelength and multi-messenger frame. The LAT survey also means variability and time-domain behavior studies [20], increased possibilities for spatial cross-match and source association studies, increased possibilities for temporal cross-correlation studies and complete, approximatively unbiased, astrophysical source population studies. The scientific return and the level of international cooperation 
is maximized studying gamma-ray photons from cosmic sources in conjunction to simultaneous data obtained from ground-based and space-borne observatories operating at other electromagnetic wavebands, or also detecting other particles (neutrinos, gravitational waves, ultra high energy cosmic rays) in the newborn multi-messenger scientific framework.

The adopted survey observational profiles during these ten years of mission were the nominal 35 degree (for the first year) or the 50 degree (for the last years) rocking angle profiles; the mixed pointing/survey profile increasing the exposure on some interesting objects (Galactic center, PSR B1259-63, the gravitational lens S3 0218+35 (lens B0218+35), the huge outburst of 3C 279, etc.) or the northern rocking (to increase exposure on the starburst galaxy M 82). Figure 1 reports the 10-year gamma-ray intensity all-sky image obtained by the Fermi LAT.

In these ten years the Fermi mission was characterized by smooth operations, with only two incidents that resulted in powering down instruments. In 2009 there was a reboot of the LAT instrument and the spacecrafts CPUs addressed with a flight software patch. On Mar. 16, 2018 the Fermi satellite encountered an issue with a drive on its $-Y$ Solar Array Drive Assembly (SADA), causing Fermi to automatically enter a"safe hold" mode, where its instruments are powered off and not acquiring science data. The LAT was powered up on Apr. 2, 2018 and allowed to reach its nominal temperature before observations resumed on Apr. 8. Since Apr. 13, 2018, the Fermi LAT operates normally again collecting science data runs.

After weeks investigating several observing modes, currently a modified rocking-angle sine profile allows the LAT to observe $80 \%$ of the sky every 1.5 hours (i.e. double of that for normal sky survey) at the cost of holes in exposure toward the sun and the anti-sun directions. This profile provides an exposure to the sky that is almost comparable to that was achieved with the 35 degrees rocking profile used during the first year of LAT operations. The NASA Flight and Science Operations teams continues to explore observing configurations to maximize the time that the 3-hour sky-survey cadence can be used within the constraints imposed by the fixed $-Y$ SADA, maintaining an almost complete sky coverage on hour timescales, with no loss of observing efficiency. In summary, although science and instrument operations were impacted for initial assessments, LAT and GBM data collection resumed quickly without major impact to the spatial all-sky survey, temporal domain monitor and serendipitous-discovery capabilities and without major impact on the overall scientific output.

After ten years of all-sky survey observations the Fermi Gamma-ray Space Telescope has largely contributed to build a new view of the structure of our Galaxy at high energies, and to shed light on the history of star formation in the Universe, while is continuing to boost the development of multi-wavelength astrophysics and time-domain astronomy. Fast transients timescales like those of Gamma-ray Bursts (GRBs) and Terrestrial Gamma-ray Flashes (TGFs) from thunderstorms on the Earth, and day-/weeks-/months-long flaring timescales of novae star systems and Active Galactic Nuclei (AGNs) take advantage of the $>10$-year LAT/GBM database of all-sky survey observations. In a similar way, the last few years have demonstrated the full power of temporal studies enabled by the Fermi LAT and GBM instruments, initiating to substantially contribute to the birth and growth of multi-messenger astro-particle physics, with the first detection of gamma-ray counterparts to both gravitational wave impulsive events and very/ultra-high energy cosmic neutrinos. A science milestone and spectacular example is the discovery of the electromagnetic gamma-ray counterpart to the gravitational wave burst GW 170817 detected by LIGO and Virgo gravitational- 
wave interferometers, and coincident with the short gamma-ray burst GRB 170817A (a binary neutron-star merger) detected by the Fermi GBM [2]. Another prime example is the discovery of the electromagnetic gamma-ray source counterpart coincident with the very high-energy neutrino event IC 170922A detected by IceCube, source that resulted to be a GeV gamma-ray active blazar, namely TXS 0506+056 (also known as ZS 0506+056, RX J0509.3+0541, MG1 J050927+0541 and 3FGL J0509.4+0541, $z=0.3367$ ), detected by the Fermi LAT [18, 1, 17].

In 10 years of operation the Fermi LAT accomplished the following record of basic statistics:

- more than 60 billion frames of science data;

- more than $40000 \mathrm{Ku}$-band antenna data telemetry passes for a total of more than 6000 hours;

- $90 \%$ of mission time spent on science sky survey;

- Autonomous Repoint Requests (ARR) for GRB triggers for a total time fraction of about $0.7 \%$

- Target of Opportunity (ToO) fixed pointed observations for a total time fraction of about $9.3 \%$;

- 821790 operational commands uplinked to the spacecraft;

- 8 releases of high level analysis software, each providing enhancements and new features;

- 4 full LAT data processing and reprocessing runs and releases, Pass 6, Pass 7, Pass 7REP and Pass 8 data;

- at least 6 people on shift every day (Flight Operations Team on duty scientist, FSSC duty scientist, GBM GRB Burst Advocate, LAT GRB Burst Advocate, LAT flare advocate (for longer duration flares/transients and all sky sources daily quick-look inspection), LAT instrument Data Quality Monitor;

- more than 3000 publications from Aug. 2008 to Aug. 2018 (where more than 100 have $>400$ citations);

- 11 years/cycles of guest investigator program with about 1500 proposers and about 220 reviewers;

- 8 international Fermi science symposia ;

- 65 press releases/features issued by NASA, most accompanied by videos or animations ( $>10$ millions views of these animations);

- 5 Bruno Rossi Prizes of the American Astronomical Society that are directly addressed to the Fermi LAT and GBM teams or to scientists that have made significant discoveries using public LAT/GBM data, and one Panofsky Prize in Experimental Particle Physics; 
- 4 general FGL source catalogs, 3 general FHL high-energy source catalogs, 1 low-energy source catalog, and more than a dozen of gamma-ray source-class specific catalogs (AGNs, GRBs, pulsars, SNRs, spatially extended sources [8, 7], all-sky variability detections [4], TGFs, solar flares);

- 7 subsequent releases of LAT background models (diffuse interstellar and isotropic models);

- new transient search software pipelines (for example for quicklook analysis of GRBs, transients and localization regions of LIGO/Virgo gravitational waves);

Beyond the statistics reported above the, so called, new Pass 8 LAT data (P8R3 is the last version, [15]), representing a major effort with full reprocessing of the entire mission dataset, is now the best high-level dataset for all types of LAT analysis. Current LAT Pass 8 data benefit from improved event reconstruction, a wider gamma-ray energy range, better energy measurements, and significantly increased effective area, resulting in sharper gamma-ray maps and larger gamma-ray count statistics, dramatically improving Fermi LAT performances. The first version of Pass 8 data was released in June 2015. Pass 8 data required a full reprocessing of raw LAT data using completely new reconstruction code that achieves better performance with a new event analysis, reconstruction and selection strategy, and following a multi-year effort. Pass 8 provides a new set of data events, Instrument Response Functions (IRFs) and analysis tools. The improvements include the pile-up handling, accidental coincidence effects "ghost events" correction, new event classes, new event types beyond the simple "front" and "back" event partition by conversion type. After ten years of Fermi mission, both the LAT and GBM instruments have better performance than ever, with enhancements to pipeline processing and analysis software, and the release of the new fourth Fermi LAT source general catalog [22]. The hardware is rather robust and not expected to degrade, the data processing and configuration is complex and tunable.

On the other hand science capabilities of the Fermi mission will continue to improve with time. The restless high-energy, MeV-GeV energy, gamma-ray sky is constantly changing, with something new to see every day, enlarging potentialities for time-domain variability studies, transients analysis, and serendipitous discoveries. For Fermi, planned as mission beyond ten years, the case for an extended mission is still valid today, with the continued detection by the LAT of gammaray photons, cosmic electrons and protons messengers, and continued operations that will enable new discovery spaces in energy, temporal (millisecond to to multi-year variability) and sensitivity domains. Fermi Galactic and extragalactic astrophysics is possible with gamma-ray pulsars, AGNs, blazars, radiogalaxies, jets, accretion processes, shocks and particle acceleration, star forming regions, high and low mass binaries, novae, gamma-ray bursts, supernova remnants, extragalactic background light, sun, moon, clusters, terrestrial gamma-ray flashes, Fermi Bubbles and unidentified sources. Fermi astro-particle and fundamental physics is possible with dark-matter WIMPs model constraints, axion like particle candidate searches, with cosmic rays acceleration sites and mechanisms, propagation physics and diffuse emission. In addition the new and incoming observatories and experiments, like LIGO/Virgo/Kagra, IceCube, KM3Net, Auger, e-ROSITA, CTA, JWST, IXPE, LSST, E-ELT, ALMA, SKA, will create new opportunities for the Fermi mission in the next years. These new multi-frequency and multi-messenger instruments provide enhanced 


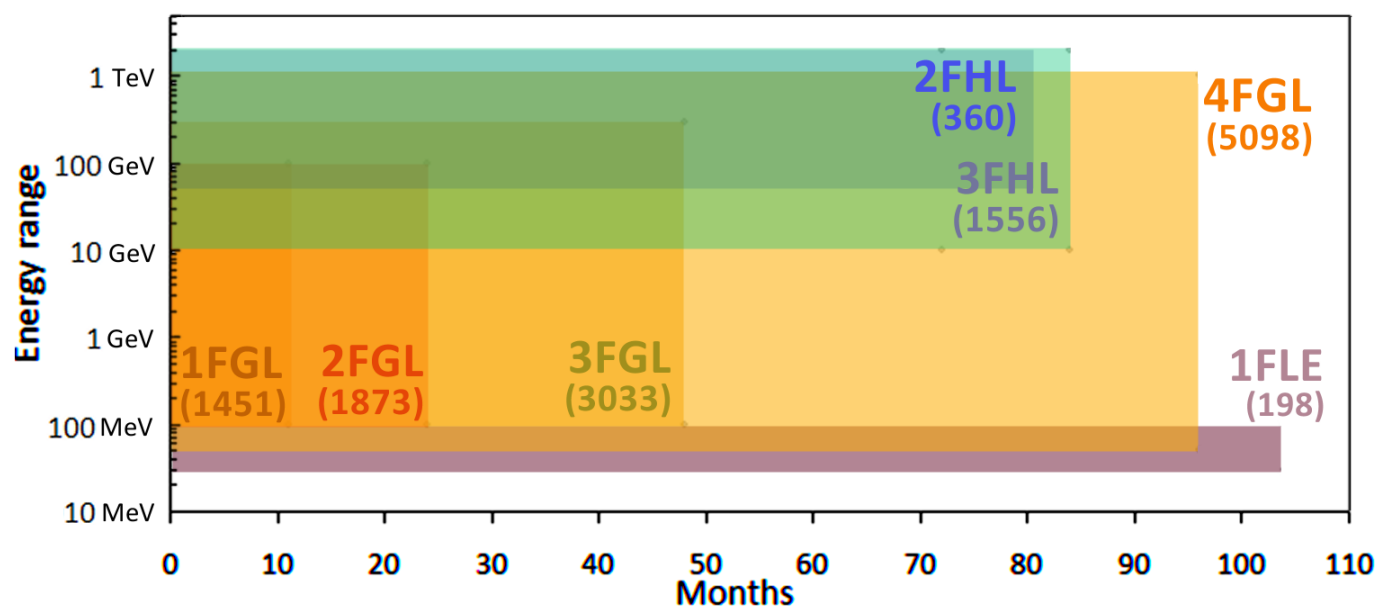

\begin{tabular}{|c|c|c|c|c|c|}
\hline 1FGL & P6_V3_DIFFUSE & gll_iem_v02 & $100 \mathrm{MeV}-100 \mathrm{GeV}$ & 11 months & 1451 point sources \\
\hline FGL & P7SOURCE_V6 & gal_2yearp7v6_v0 & $100 \mathrm{MeV}-100 \mathrm{GeV}$ & 2 years & 1873 point sources \\
\hline FGL & P7REP_SOURCE_V15 & gll_iem_v06 & $100 \mathrm{MeV}-300 \mathrm{GeV}$ & 4 years & 3033 point sources \\
\hline GES & P8R2_SOURCE_V6 & gll_iem_v06 & $10 \mathrm{GeV}-2 \mathrm{TeV}$ & 6 years & 46 extended sources \\
\hline $\mathrm{FHL}$ & P8R2_SOURCE_V6 & gll_iem_v06 & $10 \mathrm{GeV}-2 \mathrm{TeV}$ & 7 years & 1556 point sources \\
\hline HES & P8R2_SOURCE_V6 & gll_iem_v06 & $1 \mathrm{GeV}-1 \mathrm{TeV}$ & 7.5 years & 24 extended sources \\
\hline $1 / \mathrm{LL}$ & P8R2_SOURCE_V6 & & $100 \mathrm{MeV}$ & 8.75 years & 198 point sources \\
\hline r & P8R3_SOURCE_V2 & new model & $50 \mathrm{MeV}-1 \mathrm{TeV}$ & 8 years & 5098 point sources \\
\hline
\end{tabular}

Figure 2: Plot and graphic table reporting some of the published general LAT catalogs, like the three previous $\mathrm{nFGL}$, the last $\mathrm{nFHL}$ (3FHL, [10]), the two catalogs on extended sources, the low energy source catalog and the new 4FGL catalog. Point/extended source in the table refers to the catalog's objective. For catalogs references see [22] and references therein.

capability to explore Fermi gamma-ray sources, in time domain, gravitational domain and multiparticle production domain.

\section{The fourth Fermi gamma-ray LAT source catalog (4FGL)}

The Fermi LAT is by far the most sensitive detector ever in the GeV band, and it benefits from a stable response, relatively uniform sky coverage and a long baseline mission, that is optimal for building gamma-ray source catalogs. The opportunities, discoveries and main challenges for LAT catalog analysis are the same as for earlier high-energy missions (bright backgrounds, limited angular resolution and poor photon statistics). Fermi increased the number of known gamma-ray emitting objects well beyond one order of magnitude compared to previous experiments, and has added several new source classes not previously known in this gamma-ray energy range. General FGL and FHL LAT point source catalogs result from an analysis over successively deeper data sets, and represent also successive analysis refinements, from event classification on up (Figure 2).

Uniform, systematic analysis has a number of useful objectives. As mentioned in the previous section there is also a substantial number of LAT and GBM class-specific catalogs published for different types of gamma-ray sources. LAT nFGL catalogs are built to know what the LAT has detected, to set an approach for finding new gamma-ray source classes, enable studies of source 
population and to study systematically the gamma-ray sky. nFGL catalogs also represent a standard model-fitting LAT source analysis and an initial estimate for following up and detailed study of any source. The dataset of observations used to build $\mathrm{nFGL}$ and $\mathrm{nFHL}$ catalogs are nearly continuous during the Fermi LAT survey interval, although there are a few data gaps for operational issues, special calibration runs or other reasons, and there are short pointed observations and one year of modified survey profile dedicated to enhanced exposure of the Galactic center. As mentioned the current modified rocking-angle sine profile, adopted after the solar panel anomaly, results in $85 \%$ of the sky covered every 1.5 hours, giving only exposure holes in sun and anti-sun directions, but this is not significantly affecting LAT GRB detections in search for gravitational waves counterparts and, on average, over 1 year the total exposure (of interest for catalogs and other systematic studies) is very similar to the uniform all-sky survey mode of years previous to the anomaly.

The new fourth Fermi gamma-ray LAT point source (4FGL) catalog [22] represents the first census of the gamma-ray sky between $50 \mathrm{MeV}$ and $2 \mathrm{TeV}$, using the improved Pass 8 LAT data and eight years of all-sky survey observations. The 4FGL catalog is published with the release of several products like light curves and energy-band spectra of the catalog sources, with the release of the new interstellar emission and isotropic emission models, and with the publication of the companion fourth LAT AGN catalog (4LAC).

With much larger photon count statistics than the previous 3FGL catalog [9] below a few $\mathrm{GeV}$ the source detection and characterization are increasingly limited by our imperfect knowledge of the interstellar emission, which dominates the gamma-ray sky. This effect is particularly strong near the Galactic plane, but is important up to a few $100 \mathrm{MeV}$ over the entire sky. As consequence there was a long term work devoted to construct of a new model of underlying diffuse Galactic emission. An early, preliminary version of the 4FGL catalog has been released at the end of February 2019.

This superseded the FL8Y source list distributed in 2018, that was a preliminary LAT list of sources initially meant to help in writing 2018 NASA Fermi Guest Investigator proposals, and issued on January 2018. The 4FGL general catalog fully replaces this list now, and benefits from the construction of an improved diffuse emission model. The 4FGL catalog includes 5098 sources (Figure 3) above $4 \sigma$ significance, for which localization and spectral properties are provided. 75 sources are modeled explicitly as spatially extended, and overall more than 350 sources are considered as identified based on angular extent or correlated variability observed at other wavelengths. For about 1500 sources there are not plausible associated counterparts found at other wavelengths, while more than 3100 sources are associated with blazars and other AGN or candidates, and 241 sources are gamma-ray pulsars. The early 4FGL catalog does not contain all the products still (spectral energy distributions in broad bins, 2-month bin light curves, careful assessment of confused sources) and part of the associations will be updated. The 4FGL catalog builds on several generations of general nFGL, nFHL and extended sources catalogs, as reported in Figure 2.

In the 4FGL catalog only binned likelihood analysis is used because unbinned mode is much more CPU intensive, and does not support weights or energy dispersion. The 4FGL catalog [22] benefits from a number of improvements with respect to 3FGL [9], besides the twice longer exposure.

- Pass 8 data were used $[12,15]$ where the main difference relative to the P7REP data used 


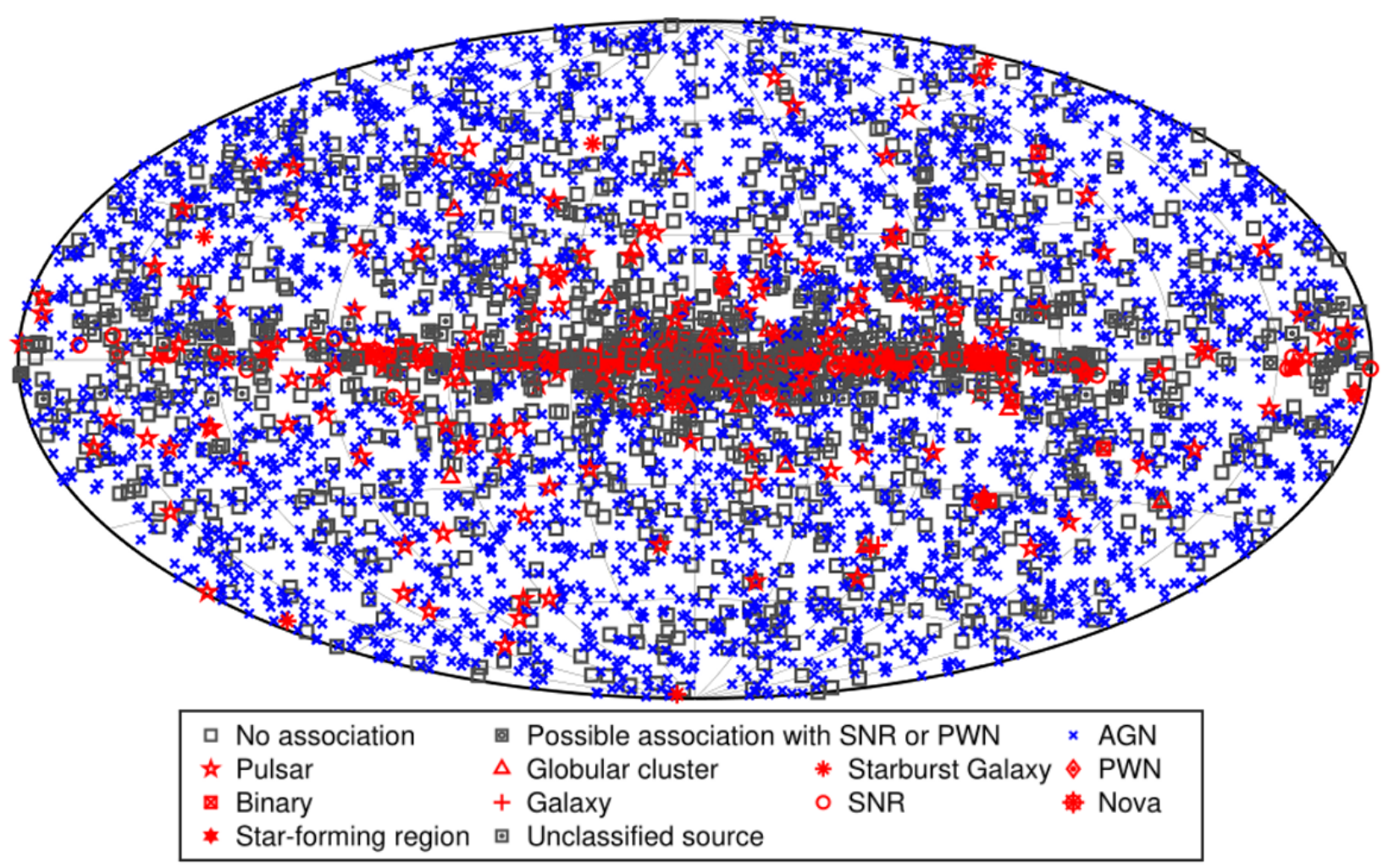

Figure 3: Pictorial all-sky map in Galactic coordinates and Hammer-Aitoff projection showing the sky loci of 4FGL catalog sources and their class. All AGN classes are plotted with the same blue symbol, other associations to a well-defined class are plotted in red, while unassociated sources and sources associated to counterparts of unknown nature are plotted in dark grey [22].

for the $3 \mathrm{FGL}$ is about $20 \%$ larger acceptance at all energies and improved angular resolution above $3 \mathrm{GeV}$.

- A new model of underlying diffuse Galactic emission was developed. The model is based on linear combinations of templates for components of the Galactic diffuse emission. For the 4FGL catalog all the templates are updated with refined partitioning the $\mathrm{HI}$ and $\mathrm{H}_{2}$ (2.6$\mathrm{mm}$ CO line) into separate ranges of Galactocentric distance ('rings'), and a new template is added for all-sky high-resolution, 21-cm spectral line HI4PI survey as tracer of HI. The interstellar emission dominates in the Galactic Ridge and the dark gas is responsible for a large part of the small-scale structures of the interstellar matter and gamma-ray diffuse emission. Recent published data are used in the new model, and improvements are reached against spurious structures around massive star-forming regions.

- Weighted logarithmic maximum likelihood analysis is adopted to mitigate the effect of systematic errors due to the imperfect knowledge of the Galactic diffuse emission.

- 75 4FGL sources are explicitly modeled as extended emission regions, up from 25 in 3FGL.

- To study the associations of LAT sources with counterparts at other wavelengths, several catalogs of counterparts at lower frequencies are updated and correspondingly the association procedure is recalibrated. 


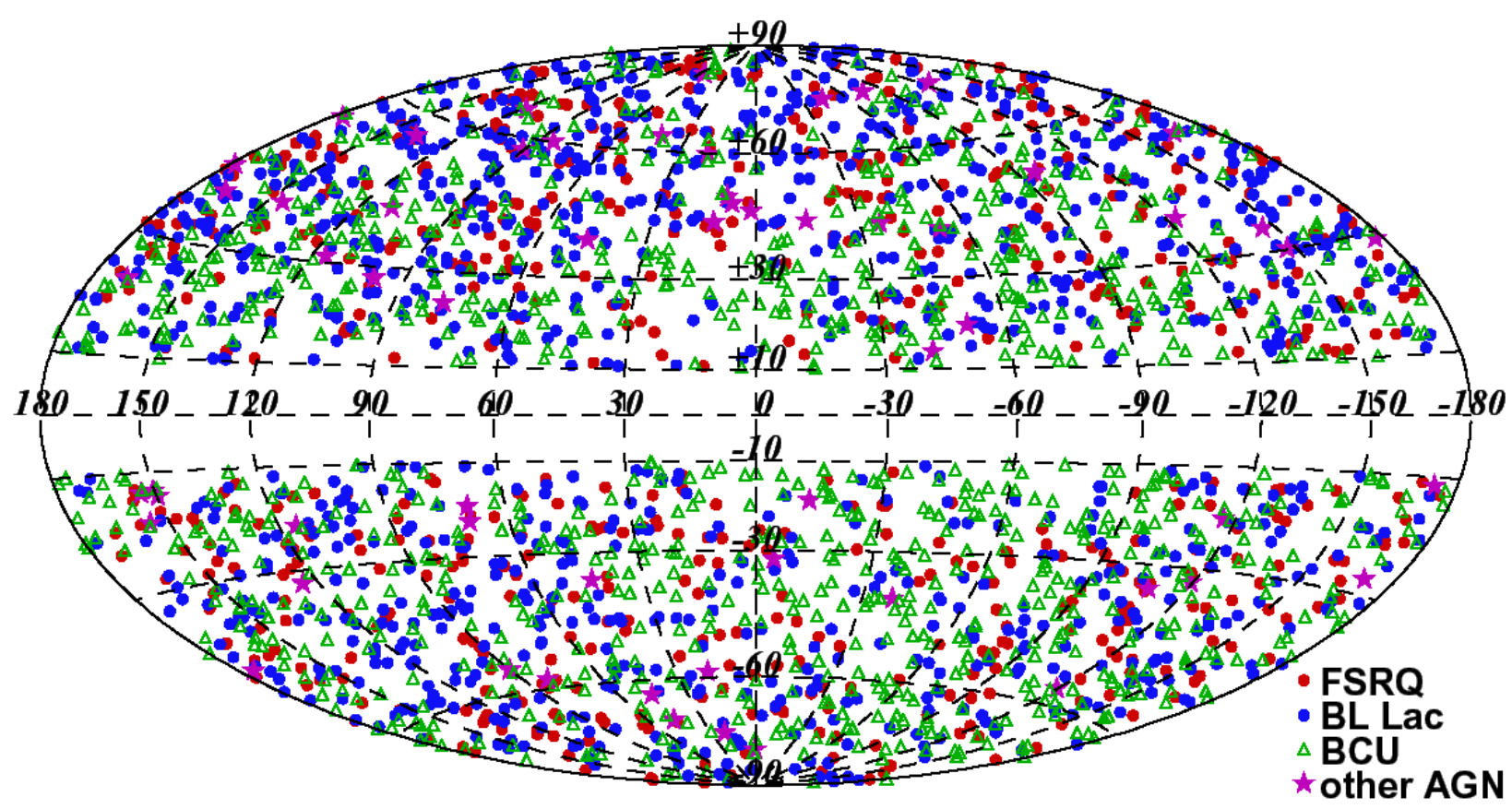

Figure 4: Pictorial all-sky map in Galactic coordinates and Hammer-Aitoff projection showing the sky loci of 4LAC catalog clan sample sources.

Also the procedure used to construct the 4FGL catalog has a number of improvements relative to that of the 3FGL catalog, improvements pertaining to detection and localization also for faint sources, significance and thresholding evaluation, Earth limb emission treatment, consideration of the effect of energy dispersion, some new parametrization of the spectral representation of sources, dedicated spatial templates for extended sources. The source detection threshold depends slightly on spectral shape. The 95\% confidence error radii for high-latitude sources $25<T S<100$ is 4.5 arcmin, about halved with respect to the first, 1FGL, catalog. The overall fraction of 4FGL sources associated to a counterpart at lower energy band is around $70 \%$, similar to that obtained in previous LAT catalogs. The association fraction is lower for fainter sources, in particular due to their larger error ellipses, while all the bright sources are associated. This fraction of associated sources also drops as sources lie closer to the Galactic plane. Even it contains more faint sources the 4FGL association rate is similar to the 3FGL, thanks to more complete catalogs and smaller error boxes. The LAT nFGL catalog analysis procedure has been applied, with successive refinements, several times and more LAT catalogs, also class-specific catalogs, will be developed in the next years.

\section{The fourth Fermi LAT AGN catalog (4LAC)}

One of the major scientific goals of the Fermi LAT is to find new sources and provide new data on the gamma-ray activity, jets, accretion processes and emission processes of gamma-ray blazar and other AGN. AGN, $\sim 10 \%$ are radio-loud, are compact objects in the gravitational center of galaxies that shows evidence for a strong non-stellar continuum emission. Such active galaxies with an AGN in their center, are believed to be well below $10 \%$ of all the galaxies in the Universe, but the percentage increases looking to higher redshifts. The companion fourth Fermi LAT AGN catalog (4LAC), preliminarily composed of more than 3000 sources, is focussed on blazars and other AGN, objects representing more than $75 \%$ of all the high-Galactic-latitude gamma-ray sources detected 
in the 4FGL catalog. A pictorial all sky map of the sky loci of 4LAC sources is reported in Figure 4. The 4LAC catalog includes information on AGN source or candidate associations, basic multifrequency knowledge, source class, synchrotron-peak parameters, redshifts, gamma-ray spectral and variability properties, and characterization of the whole gamma-ray AGN population.

The automated source association procedure for 4LAC catalog sources follows the Bayesian method adopting several lower-energy (radio, optical, X-ray) catalogs and implemented with the gtsrcid LAT software tool. It relies on the fact that the angular distance between a LAT source and a candidate counterpart is driven by the position uncertainty in the case of a real association and the counterpart density in the case of a false (random) association. In complement to this method the Likelihood-Ratio (LR) method provides supplementary associations with blazar candidates based on large, all-sky-covering, radio and X-ray surveys. The source counterpart association is like a calibrated cross correlation between source catalogs and surveys (covering the entire sky or complementary like the NVSS, SUMSS, PMN, ATCA $20 \mathrm{GHz}$, ROSAT RASS-FSC and RASS-2RXS), providing quantitative probabilities of association and false positive association rate ( 0.8 is the probability threshold adopted). A blazar or other AGN candidate is associated if the association probability is above 0.8 in one of the 2 methods. Beyond the source association the source identification is a much stronger term, that is based on observation of cross-correlated multi-frequency variability or spatial extent. There was the need to constantly improving all-sky counterpart catalogs to keep up with improving depth of the 4FGL catalog.

There are also two classification schemes for the associated blazars and other AGN:

- 1) based on the optical spectrum and the strength of broad lines (Flat Spectrum Radio Quasar FSRQs, BL Lac objects, blazar candidates of unknown type BCUs);

- 2) based on the best sampled radio-to-X-ray spectral energy distribution (SED) with algorithm and manually-controlled source by source synchrotron SED fit (low-, Intermediate-, High-synchrotron peaked, LSPs, ISPs, HSPs).

Synchrotron SED fits were performed by about 40 scientist and students of the LAT collaboration using the SSDC SED Builder tool ${ }^{5}$ and data archive. The new 4LAC blazars and other AGN associations are still under inspection regarding the optical spectrum, multi-frequency SED data, published literature, redshifts, radio and X-ray properties, to confirm their potential "blazarness".

FSRQs and BL Lacs have soft and hard gamma-ray continuous spectra respectively and the correlation between gamma-ray spectral hardness and synchrotron peak frequency appear preliminary confirmed, also for BCUs. Blazar candidates of unknown type have photon index distribution straddling the two classes. The fractions of 4LAC sources showing significant gamma-ray variability is higher for FSRQS with respect to BL Lac objects.

In conclusion the $4 \mathrm{LAC}$ catalog is a significant improvement over the previous 3LAC cata$\log$ [6] regarding analysis method and data quality. An important need is to constantly improve the all-sky counterpart (radio/optical/X-ray) catalogs to keep up with improving depth of the LAT gamma-ray catalogs and the refinements of the gamma-ray source association procedures. More details will be published in the incoming companion paper of the 4LAC catalog. A gamma-ray variability, spectral and evolutionary characterization of the detected AGN population will be included in the 4LAC paper, like implications for the BL Lac object and FSRQ dichotomy, sample biases,

\footnotetext{
5tools.ssdc.asi.it/SED/
} 
and sequence-diagram-based interpretations, information on associations, redshifts, estimation of the synchrotron SED peak and synchrotron shape parameters. This will represent a reference for studies on individual gamma-ray blazars, radiogalaxies and other AGN in the next years.

\section{Acknowledgements}

The Fermi LAT Collaboration acknowledges generous ongoing support from a number of agencies and institutes that have supported both the development and the operation of the LAT as well as scientific data analysis. These include the National Aeronautics and Space Administration and the Department of Energy in the United States, the Commissariat à l'Energie Atomique and the Centre National de la Recherche Scientifique / Institut National de Physique Nucléaire et de Physique des Particules in France, the Agenzia Spaziale Italiana and the Istituto Nazionale di Fisica Nucleare in Italy, the Ministry of Education, Culture, Sports, Science and Technology (MEXT), High Energy Accelerator Research Organization (KEK) and Japan Aerospace Exploration Agency (JAXA) in Japan, and the K. A. Wallenberg Foundation, the Swedish Research Council and the Swedish National Space Board in Sweden. Additional support for science analysis during the operations phase is gratefully acknowledged from the Istituto Nazionale di Astrofisica in Italy and the Centre National d'Études Spatiales in France.

\section{References}

[1] Aartsen, M. G., Ackermann, M., Adams, J. et al. 2018, Science, 361, eaat1378

[2] Abbott, B. P., Abbott, R., Abbott, T. D., et al. 2017, ApJL, 848, L13

[3] Abdo, A. A., Ackermann, M., Ajello, M., et al. 2009, Astropart. Phys., 32, 193

[4] Abdollahi, S., Ackermann, M., Ajello, M., et al. 2017, ApJ, 846, 34

[5] Ackermann, M., Ajello, M., Albert, A., et al. 2012, ApJS, 203, 4

[6] Ackermann, M., Ajello, M., Atwood, W. B., et al. 2015, ApJ, 810, 14

[7] Ackermann, M., Ajello, M., Baldini, L., et al. 2018, ApJS, 237, 32

[8] Ackermann, M., Ajello, M., Baldini, L., et al. 2017, ApJ, 843, 139

[9] Acero, F., Ackermann, M., Ajello, M., et al. 2015, ApJS, 218, 23

[10] Ajello, M., Atwood, W. B., Baldini, L., et al. 2017, ApJS, 232, 18

[11] Atwood, W. B., Abdo, A. A., Ackermann, M., et al. 2009, ApJ, 697, 1071

[12] Atwood, W. B., Albert, A., Baldini, L., et al. 2013, eConf C121028, arXiv:1303.3514

[13] Atwood, W. B., Bagagli, R., Baldini, L., et al. 2007, Astropart. Phys., 28, 422

[14] Bignami, G. F., Boella, G., Burger, J. J., et al. 1975, Space Science Instr., 1, 245

[15] Bruel, P., Burnett, T. H., Digel, S. W., et al. 2018, arXiv:1810.11394

[16] Fichtel, C. E., Hartman, R. C., Kniffen, D. A., et al. 1975, ApJ, 198, 163

[17] Paiano, S., Falomo, R., Treves, A., \& Scarpa, R. 2018, ApJL, 854, L32

[18] Tanaka, Y. T., Buson, S., \& Kocevski, D. 2017, ATel \#10791, 1

[19] Tavani, M., Barbiellini, G., Argan, A., et al. 2008, Nuclear Instr. Methods Phys. Res. A, 588, 52

[20] Thompson, D. 2018, Galaxies, 6, 117

[21] Thompson, D. J., Bertsch, D. L., Fichtel, C. E., et al. 1993, ApJS, 86, 629

[22] The Fermi-LAT collaboration 2019, arXiv:1902.10045 